\title{
DIE ORCHESTERWERKE ISAAC ALBÉNIZ‘ NACH DEM MANUSKRIPT 984 DER „BIBLIOTECA DE CATALUNYA“: VERSUCH EINER EINSCHÄTZUNG
}

Agustí BRUACH

\begin{abstract}
This paper mainly deals with Isaac Albéniz' orchestral music (1860-1909), often partially reviled in favor of his piano music, after Manuscript 984 from the "Biblioteca de Cataluya" (Barcelona coming to light, and having its origin in the musician's family legacy. The composer born in Camprodon appears here located in the context of the Catalan Modernism at the change of the century who is then partially influenced by the first waves of Wagnerism, from the interpretations by german composed performed in Spain, Manuscript 984 clearly shows the insuperable contradiction that the scholar has to face when considering completed works like the tone poem Catalonia (1908), with a remarkably rich orchestration reminding the French scent of the Paul Dukas school; on the other, a number of rough drafts, often written an extremely neglected scoring technique, that seem to be created by a different composer. Compositions like the aforementioned Catalonia suggest the necessity of considering the probable intervention of an orchestrator in Albéniz' works in which the orchestral palette appears with a wider range of nuances.
\end{abstract}

\begin{abstract}
Abstrakt
Der Aufsatz befaßt sich vor allem mit der orchestralen Musik Isaac Albéniz‘ (1860-1909) nach dem aus dem Nachlaß der Familie des Komponisten stammenden Manuskript 984 de „Biblioteca de Catalunya“ in Barcelona, die zu Gunsten seiner Kompositionen für Klavier oft teilweise verschmäht wurde. Der Komponist aus Camprodon erscheint hier im Kontext des katalanischen Modernismus der Jahrhundertwende, welcher wenig später mit den spanischen Erstaufführungen der Opern Wagners zumindest teilweise unter den Einfluß der ersten Wellen des Wagnerismus geriet. Das Manuskript 984 zeigt deutlich den unversöhnlichen Widerspruch, mit dem sich der Forscher bei dem Unterfangen einer wertenden Einordnung der Orchestermusik Albéniz' konfrontiert sieht: Neben vollständig auskomponierten Werken wie der symphonischen Dichtung Catalonia (1908), deren nuancenreiche Instrumentierung an die französische Schule Paul Dukas' erinnert, finden sich zahlreiche Entwürfe von häufig äußerst nachlässiger instrumentaler Technik, welche der Feder eines anderen Komponisten zu entstammen scheinen. Werke wie die oben gennante Catalonia legen für die Kompositionen, deren Orchesterpalette reichhaltige Schattierungen aufweist, die Notwendigkeit nahe, die wahrscheinliche Intervention eines anderen Komponisten bei ihrer Instrumentierung in die Erwägungen mit einzubeziehen.
\end{abstract}

\section{Albéniz und die Orchestermusik: eine problematische Frage}

Das Thema Albéniz erwies sich für den Musikologen und Musikhistoriker immer als unbequem. Wenn dies schon in Bezug auf einige Aspekte seiner Biographie oder auf seine 
Bühnenwerke gilt, nehmen die Probleme bei der Beschäftigung mit seiner Musik für Orchester noch zu. Daß sich Albéniz selbst nicht für einen Meister der Instrumentation hielt, stellt keine bedeutende Neuigkeit dar. Ganz offenkundig liegt das Niveau von Werken wie der Rapsodia Española ${ }^{1}$ für Klavier und Orchester in der Fassung von 1911, ausgeführt von Enescu, wesentlich über jenem, welches die übrigen Orchesterwerke Albéniz' generell aufweisen - mit zwei äußerst bemerkenswerten Ausnahmen: der Suite Catalonia aus dem Manuskript 984, das ich hier beschreiben will, und dem Stück El Puerto ${ }^{2}$ (Suite Iberia Nr. 2), enthalten im Manuskript 980, ebenfalls in der „Biblioteca de Catalunya“.

Meiner Meinung nach ist es zwingend, die Intervention einer zweiten Hand nicht nur bei der Vollendung der oben zitierten Werke, sondern auch bei der Oper Merlin (ca. 1906) anzuerkennen. Nämlicher Verfasser der Orchestrierung weist eindeutige Verbindungen zur Schule Paul Dukas' auf, mit welcher Albéniz immer vorzügliche Beziehungen unterhielt. Darüber hinaus neige ich zu der Ansicht, daß dieser Komponist und Instrumentateur schwerlich, wie gelegentlich vermutet wurde, mit der Figur des Mexikaners Manuel Ponce zu identifizieren ist. Wenn die Details von Ponces Biographie mit der Wirklichkeit übereinstimmen ${ }^{3}$, trat dieser nicht vor 1925 mit der Schule von Dukas in Verbindung, also sechzehn Jahre nach dem Tod Albéniz' und wenigstens zwei Jahrzehnte nach der ersten öffentlichen Aufführung der genannten Orchesterwerke Albéniz' (mit Ausnahme der Oper Merlin, die nach den mir vorliegenden Informationen noch nie in der Öffentlichkeit wiedergegeben wurde). Deshalb bleibt die Frage der Identität von Albéniz‘ Mitarbeiter bei diesen letzten Instrumentalwerken offen, wenngleich seine Intervention sich in musikalisch fundierter Weise nicht leugnen läßt.

1. „1887 beschließt Viñes, die Rapsodia española von Albéniz mit Orchesterbegleitung zu spielen, und deshalb begibt er sich zum Komponisten, um ihn um die Orchestermaterialien zu bitten:

-Er sagte zu mir, diese Rapsodia sei sehr schlecht, und er würde es vorziehen, daß ich eine spanische Suite spielte, welcher er gerade komponierte.

Vierzehn Tage darauf kehrt er zum Meister zurück, jedoch hat jener die Sätze, welche dieses Werk bilden, noch nicht fertiggestellt, eine Tatsache, aus der Viñes folgert:

-Ich hatte den Eindruck, als ob jemand seine Begeisterung für meine Fähigkeiten hätte erkalten lassen, denn er entmutigte mich im Hinblick auf meine Interpretation seines Konzerts mit Orchester, ja sogar bezüglich jeglicher Interpretation irgendeines seiner Werke!“

Zitiert nach M. Bergadà, M. Bernadó, N. Gubisch-Viñes, Ricard Viñes i Roda. Testimoni d'un temps, Lleida 1994,

S. 55

2. „Albéniz orquestó también la Evocación, y ésta junto con El Puerto se tocaron en 1907 por la orquesta de Montecarlo, que dirigía su amigo Léon Jehin. Sin embargo, ninguna certeza tenemos acerca de la interrupción de sus orquestaciones, si fue debida a la falta del tiempo necesario para escribirlas, a problemas con su ya muy quebrantada salud, $o$ a otras causas que ignoramos.

El violinista y director de orquesta Enrique Fernández Arbós, amigo íntimo de Albéniz, (...) nos da su versión: Poco después [todo parece indicar que se refiere al (...) año 1907] me escribió [Albéniz] diciendo que había instrumentado „Evocación“ y „El Puerto“; los había probado con la Orquesta de Montecarlo y aquello no iba; no sonaba. Me pedía que los instrumentase yo. Yo esperaba esa partitura para tocar „Iberia“ en Londres, por eso empecé mi orquestación de la Suite."

Zitiert nach J. Torres, Iberia de Isaac Albéniz a través de sus manuscritos, Barcelona 1998, S. 33-34

3. Siehe dazu R. Stevenson, Ponce, Manuel, in: NGrove, London 1980, Bd. XV, S. 74 


\section{Einführung: die Orchesterwerke Albéniz' im Rahmen des musikalischen Lebens um die Jahrhundertwende in Barcelona}

An der Seite seiner Klaviermusik wurden die Orchestermusik und die Bühnenwerke Albéniz‘ immer als zweitrangig angesehen. Aus historischen Gründen ist es eine Realität, daß die technischen Neuerungen, welche der neue französische Impressionismus ihnen brachte, die spanischen Komponisten des ausgehenden 19. und beginnenden 20. Jahrhunderts erst spät erreichten. Trotz der Faszination, welche die andalusische Welt auf die französischen Komponisten ausübte, trotz der traditionellen Emigration spanischer Musiker nach Frankreich erscheint es nicht zu gewagt, wenn man behauptet, der erste spanische Komponist, welcher diese Lehren inkorporiert und assimiliert hat, sei Manuel de Falla. Gerade französische Komponisten wie Fauré, d'Indy, Chausson oder Dukas konzentrieren ihre kompositorische und pädagogische Tätigkeit auf Paris, jene Stadt, die jeder der spanischen Komponisten aus dieser Zeit früher oder später aufsuchen wird.

Die Biographie Albéniz' selbst, voll von abenteuerlichen Jugendepisoden ${ }^{4}$, ist zum Teil Schuld daran, daß die Rezeption ${ }^{5}$ des Komponisten mehr mit einem Salonmusiker zu tun hat als mit einem selbständigen und der Epoche, welcher er angehört, eigenen Musiker. In den bürgerlichen Salons im Barcelona der Jahrhundertwende, zutiefst geprägt von den ästhetischen Ideen des Modernismus und zugleich von der „Renaixença“ genannten politischen und poetischen Bewegung, präsentieren sich Komponisten wie Albéniz oder Granados als authentische Virtuosen ihres Instruments ${ }^{6}$, aber auch als Meister der Komposition von Formen geringen Ausmaßes, ein Gebiet, auf welchem sie sich als wahrhaft heimisch erweisen.

Andererseits bewirkte das Eintreffen der ersten Schübe des Wagnerismus in Barcelona und später in ganz Spanien, daß sich das Interesse nunmehr der Orchestermusik im Allgemeinen und der szenischen Musik im Besonderen zuwendete. Die Opern Wagners waren generell von so entscheidendem Einfluß auf das Musikleben Barcelonas, daß Wagners Musik zu einem Synonym für Modernität und zum Inbegriff nationaler Behauptung wurde. Von der ersten vollständigen Aufführung Lohengrins 1882 in Barcelona an bis zum Jahr 1910, als Der Ring des Nibelungen

4. Die erste Biographie wurde von Antonio Guerra y Alarcón 1886 geschrieben und veröffentlicht [Isaac Albéniz, notas crítico-biográficas de tan eminente pianista (Escuela tipográfica del hospicio)].

Siehe dazu: Walter Aaron Clark, Albéniz en Leipzig y Bruselas: nuevas luces sobre una vieja historia, in: Revista de Musicología XIV Nr. 5, 1-2, 1991, S. 213-214. Jedoch weist Clark zu Recht auf die unvermeidlichen „Beschönigungen“ hin, die Albéniz an seiner Vita vornimmt: Die gesamte Ausbildungs- und Studienzeit des Komponisten müsse daher von neuem erforscht und geschrieben werden.

5. „Albéniz llevó una vida itinerante, y en varias temporadas estableció su residencia en más de media docena de ciudades de Europa, primero como intérprete virtuoso y luego como acreditado compositor. Este ingrediente nómada de su biografía, unido al escaso cuidado y control que ejerció sobre su copiosa producción musical y a la fragmentación sufrida después por las diferentes donaciones y repartos de sus herederos, ha dado lugar a una colección documental dispersa, difícilmente localizable y cuyos documentos originales se han perdido en buena parte."

J. Torres, Iberia de Isaac Albéniz..., S. 9

6. „Sociedades de conciertos, los artistas y virtuosos de más fama, tenían indicado el nombre de nuestra ciudad en su itinerario de excursiones. Se sucedían sin interrupción exposiciones artísticas, cursos, conferencias y toda suerte de manifestaciones culturales."

F. Curet, El arte dramático en el resurgir de Cataluña, Barcelona um 1918, S. 289 
unter der musikalischen Leitung von Franz Beidler dort erstmals komplett aufgeführt wurde, wuchs dieser Einfluß beständig. Dabei fand eine zunehmende Identifizierung mit der bürgerlichen Klasse statt, welche sich in der Gründung der ersten Wagnergesellschaft etwa ab 1880 niederschlug. ${ }^{7}$ Ein weiterer „deutscher“ Komponist, welcher Einfluß auf das musikalische Leben in Spanien ausübte, war Franz Liszt ${ }^{8}$, der für Albéniz ein Vorbild als Klaviervirtuose war. ${ }^{9}$ Neben der Klaviermusik waren seine zwei Symphonien von besonderer Bedeutung für die formale und instrumentale Konzipierung der symphonischen Musik durch die spanischen Komponisten der Jahrhundertwende, wobei sie sowohl aufgrund ihrer zyklischen Form als auch wegen der von Liszt gewählten literarischen Themen die späten Werke Albeniz ‘ und Granados“ entscheidend beeinflußten.

Von besonderer Bedeutung sind aus dieser Sicht auch die vier Jahre, die Albéniz in England verbrachte. Unter dem Einfluß von seinem Mäzen Francis Burdett Money-Coutts komponierte er ab dieser Zeit Vertonungen ausgewählter Texte des englischen Bankiers. ${ }^{10}$ Aber noch wichtiger wurde die Konzerttournée von 1879, in der er Abschnitte aus Opern Wagners am Klavier wiedergab:

„(...) Señor Albéniz, after playing Brassin's transcriptions of the rainbow scene from Das Rheingold and the fire charm from Die Walküre, had a final tremendous wrestle with the Walkürenritt. The dead silence produced by his playing, particular during the second piece, was the highest compliment he could have desired. (...)“11

7. Siehe dazu: Revista Musical Catalana, Juni 1913

„El drama musical de Wagner triunfaba ruidosamente en nuestro Gran Teatro del Liceo, creando una numerosa falange de devotos que luego tuvo su sede en la Associació Wagneriana, otra de las entidades que han contribuído a elevar el nivel cultural de nuestro pueblo."

F. Curet, El arte dramático..., S. 289

8. „(...) so hat sich in Spanien eine Formation von Komponisten herausgebildet (...). Besonders hervorzuheben unter ihnen der Name von Isaac Albéniz. (...) Ohne in irgend einer Beziehung Liszt zu ähneln, erinnert er mit seinem Ideenreichtum an ihn."

C. Debussy, Monsieur croche et autres écrits, Paris 1971, S. $244 \mathrm{ff}$.

9. Siehe dazu: Anmerkung Nr. 4. Vermutlich hat sich Albéniz mit Liszt in Weimar irgendwann zwischen 1878 und 1880 getroffen. In Bezug darauf findet sich eine Bemerkung im persönlichen Tagebuch Albéniz“ (Isaac Albéniz, Impresiones y Diarios de Viaje, hrsg. von E. Franco, Madrid 1990, S. 24-34; zitiert auch in: M. Raux-Deledique, Albéniz, su vida inquieta y ardorosa, Buenos Aires 1950, S. 170-179), welche in diese Richtung deutet, wenn sie sich auch mit keinem anderen zeitgenössischen Zeugen abgleichen läßt. Es könnte sich daher um eine Erfindung Albéniz" selbst handeln, welche dazu dienen sollte, seine Autobiographie selbst zu bestimmen.

10. „El banquero Money-Coutts, que gustaba de hacer pinitos literarios con el seudónimo de Montjoui, entró en relaciones con Isaac Albéniz a quién le hizo una proposición realmente tentadora. Consistía ésta en que Albéniz le diera la exclusiva de su música en el género del teatro para las obras literarias que él pudiera hacer (...). El músico llamaba a esto graciosamente el pacto de Fausto."

Antonio de las Heras, Vida de Albéniz, Madrid und Barcelona 1941, S. 92 London 1981, S. 868

11. Bernard Shaw, Shaw's Music, the complete musical criticism of Bernard Shaw, hrsg. von D. H. Laurence,

„(...) este hombre que sólo vivió cuarenta y nueve años (1860-1909), fue considerado, en sus comienzos y por casi todos, como un pianista virtuoso, un artista para exhibirse, no por la calidad de la música que compusiera o interpretase, sino por el artificio de su interpretación aunque, poco a poco y con dificultades y deficiencias que lo breve de su vida no permitieron corregir y a pesar de todo ello, llegó a adquirir una merveilleuse connaissance du métier de compositeur tal como supo reconocer el genio de Debussy. Y en la base de este conocimiento estaba la mélancolie nombreuse y el humor spécial de su país de origen y, junto con ellos, el haber sabido beber y oír sin copiar, la substancia de las músicas populares (...).“

Zitiert nach J. Soler, Vorwort zu Albéniz“ Pepita Jiménez. Comedia Lírica en dos actos, revidiert und herausgegeben von J. Soler, Madrid 1996, S. XI 


\section{Das Manuskript 984 der „Biblioteca de Catalunya“: Klaviermusik versus Orchestermusik}

Das räpresentativste Werk dieses Manuskriptes ist ohne Zweifel die ursprünglich als Suite konzipierte Catalonia, die in Paris 1899 uraufgeführt und anschließend als Einzelstück beim Pariser Verlag Durand 1908 publiziert wurde. Das erste Fragment ist im Manuskript unvollendet, wobei es sich mit großer Wahrscheinlichkeit um eine für die Uraufführung bestimmte Kopie handelt. Es sind noch kleine Markierungen mit Bleistift zu finden, und die Orchestrierung ist praktisch identisch mit der Partitur, die neun Jahre später veröffentlicht wurde. Nur kleine Bemerkungen fehlen in der ersten Version: z. B. findet sich in der veröffentlichten Version unmittelbar nach Ziffer 16 die Bezeichnung ,pavillon en l'air“ in den Hörnern, welche im Manuskript fehlt. Einen Takt nach Ziffer 25 endet die erste handschriftliche Fassung unvermittelt.

Einen sehr wichtigen Unterschied zwischen der ersten unvollständigen und der zweiten Fassung der Suite Catalonia bildet die Tatsache, daß unter dieser zweiten Version immer die Klavierfassung zu finden ist, als ob sie dem möglichen Verfasser der Instrumentierung als Grundlage gedient hätte. Diese zweite im Prinzip vollständige Handschrift weist bis zwei Takte nach Ziffer 25 keine großen Unterschiede zur Ausgabe von Durand auf (außer in der Verteilung der Harfenpartien und in den Verdoppelungen zwei Takte nach Ziffer 24, die im Originalmanuskript die Flöte und in der gedruckten Fassung die Piccoloflöte ausführt). Ab dort enthält die Durand-Ausgabe eine viel längere Durchführung des katalanischen Volksliedes (Un pobre pagès tenia una filla...), welches als Grundlage für dieses Fragment dient. Die handschriftliche Fassung, die wahrscheinlich bei der Aufführung in Paris im Jahre 1899 verwendet wurde, bricht wenig später abrupt $a b^{12}$.

Höchstwahrscheinlich war der Komponist mit der Originallänge des Satzes unzufrieden und dürfte später den ganzen neuen Abschnitt hinzugefügt haben, der in der gedruckten Ausgabe von 1906 erscheint. Dieser neue Teil umfaßt Passagen von großer instrumenteller Originalität, wie jene ab Ziffer 47 (Musiciens ambulants), in der die Klarinette, zwei Trompeten, die dritte Posaune, die Becken, die Trommel und der erste Kontrabaß die oben erwähnte Melodie in fragmentarischer Weise und mit einem extrem raffinierten Klangsinn ausführen.

Es ist darauf hinzuweisen, daß die Benutzung folkloristischer Lieder oder Themen in den Stücken Albéniz' nur ganz selten identifizierbar ist. Gleichzeitig zeigt sich jedoch die Musik des spanischen Komponisten von den neuen Sammlungen spanischer Lieder oft direkt inspiriert: Zwar kann man seine Werke nicht ohne weiteres als Vertonungen populärer Melodien bezeichnen, aber ein direkter Einfluß ist mit Bestimmtheit feststellbar. Viele der Stücke seines Meisterwerkes Iberia sind stark von den verschiedenen Arten des flamenco-Gesangs geprägt. Echte Zitate finden sich aber kaum, weder im Bereich der Melodie, noch in den typisch fixierten harmonischen Passagen der andalusischen Zigeunermusik: Es handelt sich mehr um eine 
Begabung, um eine Neigung, der die Quellen der alten spanischen folkloristischen Musik dienen und denen der Komponist ein bestimmtes, ganz persönliches Kolorit entnimmt. In diesem Zusammenhang spielt sicherlich auch das zunehmende Interesse der spanischen Historiker bzw. Musikologen an der alten volkstümlichen Musik eine entscheidende Rolle. Unter diesem Aspekt darf die zentrale Figur Pedrells nicht vergessen werden: Vor allem in seinem musikalischen Pamphlet Por nuestra música (1891) hat er die Erforschung der alten folkloristischen Quellen verteidigt als nicht nur melodisches, sondern auch ideologisches Fundament der neuen spanischen nationalen Schule. ${ }^{13}$

Aus dieser Perspektive erscheint es nachvollziehbar, daß der Komponist von den neuen Forschungen der populären Musik als Künstler stark beeinflußt war. Nach einem Hinweis des Albénizspezialisten Jacinto Torres finden sich noch heute zahlreiche Volksliedsammlungen in der ehemaligen Bibliothek des Komponisten. ${ }^{14}$

Die beiden Fassungen des zweiten Stücks aus der Suite, die sich in der Handschrift anschließen und dem Maler Josep Maria Sert (Bühnenbildner bei der Uraufführung der Josefslegende von Richard Strauss/Hugo von Hoffmannsthal an der Pariser Oper 1914) gewidmet sind, stellen nichts als reine Entwürfe dar, deren erster instrumental um ein Bratschensolo herum angelegt ist. Der zweite beinhaltet eine Harfenbegleitung, welche noch unfertiger erscheint als der erste.

Diese beiden Fragmente zusammen mit der darauffolgenden Rapsodia Almogávar zeigen ganz deutlich die Grenzen von Albéniz als Komponist für großes Orchester. Die Tatsache, daß sie die Inschrift und die Adresse des Verlags Durand über dem linken Teil von Folio 39 tragen, zeigt eindeutig die ursprüngliche Intention des Komponisten, diese Stücke zusammen herauszugeben. Die Gründe, die dies verhinderten, wird man niemals mit Sicherheit kennen, aber jedenfalls zeigt der Vergleich dieser drei letzten Projekte des Manuskripts mit der gedruckten Fassung der Nr. 1 der Suite Catalonia klar die Intervention einer anderen Hand als Hilfestellung bei den Orchesterparts.

Das folgende Stück für großes Orchester ist die symphonische Dichtung ${ }^{15}$ Lo Llacsó über einen Text des katalanischen Dichters und Liedkomponisten Apel·les Mestres. Von diesem Werk

13. „Posee España una mina musical inagotable de cantos populares de variadas procedencias. Pero ni Europa ni la mayoría de los mismos músicos españoles tienen idea cabal de la riqueza de formas directas que ofrece nuestra música popular, ni pueden adivinar, sin estudiarlas muy á fondo, la importancia de esa infinidad de melodías primitivas de tan fecunda melopea y ritmopea que brotan de todas las provincias de España y forman regiones musicales distintas y características, las cuales haría resaltar y precisaría una filología-musical, no tan precisa como la del lenguaje, pero no menos interesante para la buena dirección y fines de los importantes estudios folk-lóricos [sic].“

F. Pedrell, Por nuestra música/Algunas observaciones sobre la magna cuestión de una escuela lírico nacional, Barcelona 1891, S. 42

14. „(...) entre los libros de su biblioteca personal que se conservaban en la casa barcelonesa de sus descendientes tuve ocasión de ver la colección de Cantos populares de Galicia, Asturias y Valencia, de José Hurtado, así como las Cançons Populars Catalanas [sic] recopiladas por Francisco Alió, las Jotas aragonesas de José María Alvira y los Trente-trois chants populaires de l'Espagne de Édouard Moulle; por otra parte, un recibo de su editor Juan Bautista Pujol, fechado el 31 de mayo de 1902, acredita con la firma del propio Albéniz la adquisición de las Sevillanas de la colección de Ecos de España y un ejemplar de los Cantos y bailes populares de España de José Inzenga."

J. Torres, Iberia de Isaac Albéniz..., S. 27-28

15. Die Gattungsbezeichnung stammt vom Komponisten ungeachtet der Einbeziehung von Gesangspartien. 
sind etwa zehn äußerst einfach instrumentierte Takte erhalten. Noch öfter hat Enric Granados um die Jahrhundertwende Texte von diesem Dichter des Modernismus übernommen, in denen eine gewissermaßen simplifizierende mittelalterliche Tenden $\mathrm{z}^{16} \mathrm{zu}$ beobachten ist, die auch mit dem neuen, aus einer nationalistischen Perspektive heraus interpretierten Wagnerismus zu tun hat. Lieder wie La cançó del Gener, Cançoneta, La boira, Liliana oder Werke größerer Dimensionen wie Petrarca (1900), Picarol (um 1901), Follet (1903) und Gaziel (1906) ${ }^{17}$ bilden nur Beispiele dafür, wie sich die spanischen Komponisten dieser Zeit mit der Übernahme der neuen Tendenzen aus der mitteleuropäischen Musik beschäftigt und sich wechselseitig beeinflußt haben.

Unmittelbar im Anschluß daran findet sich eine Zarzuela en verso in beträchtlich fortgeschrittenerem Stadium. Darüber hinaus weist La real hembra eine im Vergleich zu dem soeben zitierten Werk stärker ausgearbeitete Instrumentierung auf, ohne im Hinblick auf die Kunstfertigkeit und innere Differenzierung jemals auch nur annähernd an das Niveau der ersten Nummer aus der Suite Catalonia heranzureichen. Es handelt sich um ein Werk, dessen brillante Passagen oft exzessiv, zu dick instrumentiert sind. Bei der Analyse der Folios 72 und 73 des Manuskripts sieht man etwa, wie der Komponist systematisch die Streicher in den Holzbläsern verdoppelt, wodurch die Instrumentierung gleichsam zu ersticken droht.

Das folgende Fragment ist ein Opernprojekt über Mar y cel (sic) des Dramatikers Àngel Guimerà, von dem etwa fünfzig vollständig instrumentierte Takte erhalten sind. Von neuem begegnet man der beschränkten Instrumentierkunst Albéniz‘. Die gewichtigsten musikalischen Ideen werden oft in systematischer Art und Weise von den Streichern getragen. Die Bläser beschränken sich praktisch entweder auf die Verdoppelung oder auf eine ornamentale Funktion und werden gelegentlich in Lagen benutzt, für die sie schlechterdings ungeeignet sind.

Hieran schließt sich die symphonische Suite The Alhambra nach Texten des englischen Mäzens F. B. Money-Coutts an. Von diesem Projekt begann Albéniz schließlich nur das Stück Nr. 3, El Generalife, von welchem 26 Takte erhalten sind, sowie das Stück Nr. 1, La Vega, von dem noch etwa 90 Takte existieren.

La Morena war ursprünglich als lyrisches Drama in vier Akten nach einem Text von Alfred Mortier geplant. Von ihm sind nur noch 22 Takte überliefert, in sehr embryonalem Zustand und wahrscheinlich in großer Eile ausgeführt.

Guajira sollte die Orchestrierung eines kubanischen Volksliedes für einen solistischen Gesangspart und Orchester bilden. Hiervon sind nur 2 Takte erhalten.

Das letzte Werk der Handschrift ist das lyrische Drama La Serenade. Albéniz verwendet eine Orchestrierung mit geteilten Streichern mit Dämpfer im Flageolett. Diesmal imitiert er nicht

16. Die beiden großen Dichtungen Jacint Verdaguers über Phantasiethemen sind für diese mittelalterliche Tendenz als modellhaft anzusehen: L'Atlàntida (um 1876-77) und Canigó (1886).

17. E. Granados, Integral de l'obra per a veu i piano, revidiert und herausgegeben von M. García Morante, Barcelona 1996.

„Si algo puede consolarnos y resacirnos de los desgraciados intentos de teatro lírico realizados hasta ahora este algo lo debemos a Apeles Mestres quien, gracias a aquellas efímeras pruebas, aportó a la escena catalana su maravilloso talento, inagotable inspiración poética y extremado buen gusto."

F. Curet, El arte dramático..., S. 355. 
die klassischeren Instrumentierungsmodelle der Schola Cantorum, sondern die klanglichen Neuerungen Debussys wie in Pélleas et Mélisande.

\section{Gewicht der Orchestermusik Albéniz' in seinem Gesamtwerk}

Trotz des wiederholten Versuchs, einen eigenen Orchesterstil zu schaffen, trotz eines musikalischen Denkens, welches oft über den häufig wiederholten Topos des Pianismus Albéniz“ hinausreicht, ist es dem spanischen Komponisten kaum gelungen, sein Metier im Bereich der Instrumentierung zu verbessern. Meiner Ansicht nach muß man das Problem im Zusammenhang mit der traditionellen Isolierung der iberischen Halbinsel verstehen. Der unvermeidliche soziale und politische Niedergang, den das ganze 19. Jahrhundert bildet, hat selbstverständlich seine Folgen im künstlerischen Bereich. Die verspätete Ankunft der industriellen Revolution in Spanien in der ersten Hälfte des Jahrhunderts bewirkt, daß ein durch den fortschreitenden Verlust der Kolonien immer stärker verarmtes Bürgertum sich den Luxus, Musik für große Besetzung und von großen formalen Dimensionen in Auftrag zu geben, nicht erlauben kann. Der Zeitabschnitt von 1800 - 1875 ist einer der ärmsten in der spanischen Musik und umfaßt neben einer zweitrangigen Salonmusik nur den immer größeren Erfolg der Zarzuelas auf volkstümlichem Niveau.

Es ist klar, daß mit diesem Substrat Komponisten vom Talent Albéniz' und Granados` notwendigerweise erst spät zu den klanglichen Möglichkeiten gelangen konnten, die in diesen Jahren das Orchester für einen Komponisten der französischen oder deutschen Tradition bot. Tatsächlich sind diese beiden Komponisten praktisch die ersten Repräsentanten einer über die Landesgrenzen hinaus wirkenden spätromantischen spanischen Musik, sei es als Klaviervirtuosen, sei es als Komponisten von wohlverdienter Reputation. Trotz des kollegialen Verhältnisses, das die beiden vor allem zu Komponisten des französischen Bereichs unterhielten, konnten sie nicht mehr sein als privilegierte Beobachter der letzten harmonischen Neuerungen des Wagnerismus und der Erweiterung der Schattierungsmöglichkeiten der französischen orchestralen Palette.

Es ist unmöglich zu wissen, ob Albéniz sich bei einer besseren Orchesterkenntnis als der, über welche er tatsächlich verfügte, dazu entschlossen hätte, Iberia vollständig zu instrumentieren - zweifellos einer der vollendetsten Klavierzyklen der südeuropäischen Musik der zweiten Hälfte des 19. Jahrhunderts. Dieses Wunder des Klavieres - nach dem Urteil Olivier Messiaens - geht über die dem Klavier eigentümliche Sprache hinaus und verlangt eine Anzahl und Qualität von Schattierungen, welche auf diesem Instrument beinahe unausführbar sind.

Albéniz hatte zu seiner Zeit nicht das Glück, das die Musikgeschichte für andere große Musiker mit ähnlichen Schwierigkeiten bereithielt: Mussorgski und die Bilder einer Ausstellung zeigen in äußerst beredter Art und Weise, wie eine Musik von radikal anderer, Albéniz geradezu entgegengesetzter pianistischer Textur mit Hilfe einer orchestralen Sprache zu einer neuen Dimension gelangen kann. 
Die Orchesterwerke Isaac Albéniz“ nach dem Manuskript 984 der „Biblioteca de Catalunya“

Wie man vom dargelegten Standpunkt aus ein instrumental so vollendetes Werk wie die Nr. 1 der Suite Catalonia verstehen kann, ist eine Frage, die momentan offen bleibt. Es ist klar, daß die brillante Instrumentation dieses Werkes in letzter Instanz die ihm eigene musikalische Sprache des Komponisten begünstigt. Weit davon entfernt, hieran Anstoß zu nehmen, ist vielmehr in Erinnerung zu rufen, daß Albéniz selbst deutlich genug seine Limitationen in dieser Hinsicht anerkannte, eine Tatsache, welche mitnichten die Größe seiner musikalischen Inspiration und noch weniger seiner künstlerischen Integrität mindert.

\section{Inhalt des Manuskripts 984 der „Biblioteca de Catalunya“, Nachlaß der Familie des Komponisten (1927). Instrumente in der Reihenfolge der Partitur argegeben.}

[Leider wurden die Ränder des Manuskriptes kurz nach der Lieferung an die „Biblioteca de Catalunya“ beschnitten, so daß einige Anmerkungen nicht mehr lesbar sind. Das Heft ist heute $40 \mathrm{~cm}$ breit und $27,5 \mathrm{~cm}$ hoch.]

1. (Fol. 1) Catalonia. Suite Populaire [Paris, Januar 1899, Orchesterpartitur, unvollendet, „à Ramon Casas“; Picc/2 Fl/2 Ob/E. Hrn/2 Kl in B/Bkl in B/3 Fag/4 Hrn in F/4 Tr in C/3 $\mathrm{Pos} / \mathrm{Tb} / \mathrm{Pk} / \mathrm{Tri} / \mathrm{Gr}$ Trom/Bek/Trom/2 Hrf/1, 2Vl/Br/Vlc/Kb]

2. (Fol. 10) Catalonia. Suitte (sic) populaire, Nr. 1 [Paris, April 1899, signiert auf dem Fol. 38 „Paris Auteuil/22 Avril 1899/Isaac Albéniz“, Orchesterpartitur, erste Version für die Uraufführung in Paris 1899 ?; Picc/2 Fl/2 Ob/E. Hrn/2 Kl in B/Bkl in B/2 Fag/Kfag/4 Hrn in F/4 $\mathrm{Tr}$ in C/3 Pos/Tb/Pk/Tri/Gr Trom/Bek/Trom/2 Hrf/1, 2 Vl/Br/Vlc/Kb]

3. (Fol. 39) Catalonia, Suitte (sic) Populaire, Nr. 2 [Paris, April 1899, Orchesterpartitur, unvollendet, ,à J. M. Sert“; Picc/2 Fl/2 Ob/E. Hrn/2 Kl in B/Bkl in B/3 Fag/4 Hrn in F/4 Tr in $\mathrm{C} / 3 \mathrm{Pos} / \mathrm{Tb} / \mathrm{Pk} / 2 \mathrm{Hrf} / 1,2 \mathrm{Vl} / \mathrm{Br}$ (1 Br solo)/Vlc/Kb]

4. (Fol. 46) Catalonia, Suitte (sic) Populaire, Nr. 2 [zweite Version, Orchesterpartitur, unvollendet, ,à J. M. Sert“; Picc/2 Fl/2 Ob/E. Hrn/2 Kl in B/Bkl in B/3 ? Fag/4 Hrn in F/4 Tr in $\mathrm{C} / 3 \mathrm{Pos} / \mathrm{Tb} / \mathrm{Pk}$ in $\mathrm{B}-\mathrm{Es} / 2 \mathrm{Hrf} / 1,2 \mathrm{Vl} / \mathrm{Br} / \mathrm{Vlc} / \mathrm{Kb}]$

5. (Fol. 58) Aventura de los molinos [undatiert, Orchesterpartitur, Frag.; Picc/2 Fl/2 Ob/E. $\mathrm{Hrn} / 2 \mathrm{Kl}$ in B/Bklar in B/3 Fag/4 Hrn in F/4 Tr in C/3 Pos/Tb/Pk D-G/Gr Trom/Trom/Tri/2 Hrf/1, $2 \mathrm{Vl} / \mathrm{Br} / \mathrm{Vlc} / \mathrm{Kb}]$

6. (Fol. 61) Rapsodia Almogavar (sic) [Januar 1899, Orchesterpartitur, Frag. der Suite Catalonia?; Picc/2 Fl/2 Ob/E. Hrn/2 Kl in B/Bkl in B/3 Fag/4 Hrn in F/4 Tr in C/3 Pos/Pk/Gr Trom/Trom/2 Hrf/1, 2Vl/Br/Vlc/Kb]

7. (Fol. 62) Conseil tenu par les rats [undatiert, unsigniert, für eine Singst. und Klavier, Frag.]

8. (Fol. 63) Lo llacsó/Poema Sinfonico/en un /Prologo/y/Tres actos/para Orquesta, Coros, Soprano, Mezo, Tenor y Baritono/Poema de Apeles Mestres/musica de I. Albeniz [Paris, 1. April 1896, Orchesterpartitur, Frag.; Lo vent, Bariton/Una llevor de Llacsó, Tenor/Una Papellona, Sopran/Una Abella, Mezo (sic)/Coro de hombres y mujeres; Picc/2 Fl/2 Ob/E. Hrn/K1 in B/2 Bkl in $\mathrm{B} / 2$ ? Fag/Kfag/4 Hrn in $\mathrm{F} /$ ? Tr in $\mathrm{B} / 3 \mathrm{Pos} / \mathrm{Tb} / \mathrm{Pk} / 1,2 \mathrm{Vl} / \mathrm{Br} / \mathrm{Vlc} / \mathrm{Kb}]$ 
9. (Fol. 66) La Real Hembra/Zarzuela en verso [Libr. von Cristóbal Castro, Madrid, September 1902; Präludium, 1. und 2. Szene; 3. Szene unvollendet, Orchesterpartitur; Joselillo/Antoñico/Carmen; Picc/2 ? Fl/2 ? Ob/E. Hrn/2 Kl in B/Bkl in B/2 Fag/4 Hrn in F/2 Tr in $\mathrm{B} / 3 \mathrm{Pos} / \mathrm{Tub} / \mathrm{Pk} \mathrm{B}-\mathrm{Es} / \mathrm{Hrf} / 1,2 \mathrm{Vl} / \mathrm{Br} / \mathrm{Vlc} / \mathrm{Kb}]$

10. (Fol. 93) Mar y Cel (sic) [undatiert, unsigniert, Projekt der Oper über das Theaterstück von Àngel Guimerà, Orchesterpartitur, Frag.; Osman/Mahomet/Joanot/Hassen/ Chor; Picc/2 Fl/2 Ob/E. Hrn/2 Kl in A/Bkl (in B)/2 Fag/Kfag/4 Hrn in F/? Tr in F/3 Pos/Tb/Pk $\mathrm{H}-\mathrm{E}$ ?/1, $2 \mathrm{Vl} / \mathrm{Br} / \mathrm{Vlc} / \mathrm{Kb}]$

11. (Fol. 102) The Alhambra/Suite Symphonique/sur le Poeme/de/F. B. Money Coutts/Musique de/I. Albeniz [Paris, Februar 1897, Nr. 1, La Vega, Nr. 3, Generalife, Orchesterpartitur, beide unvollendet; Picc/2 Fl/2 Ob/E. Hrn/2 Kl in B/Bkl in B/2 Fag/Kfag/4 Hrn in $\mathrm{F} /$ ? $\mathrm{Tr}$ in $\mathrm{F} / 3 \mathrm{Pos} / \mathrm{Tb} / \mathrm{Pk}$ Des-As/Hrf/1, 2Vl/Br/Vlc/Kb]

12. (Fol. 115) La Morena/Drame Liriquelen quatre actes/parolles d'Alfred Mortier/Musique d'I. Albeniz [Nice, 21 April 1905, Orchesterpartitur, unvollendet; Picc/2 Fl/? Ob/E. Hrn/2 Kl ?/Bkl ?/2 Fag/? Hrn/? Tr/? Pos/Pk/2 ? Hrf/1, 2 Vl/Br (Br solo)/Vlc/Kb]

13. (Fol. 118) Guajira/(Chant populaire Cubain)/Repertoire des concerts de Maria Gay/par I. Albeniz [Brüssel, Februar 1905, Orchesterpartitur, Frag.; Picc/2 Fl/2 Ob/E. Hrn/2 Kl in $\mathrm{B} / \mathrm{Bkl}$ in $\mathrm{B} / 2 \mathrm{Fag} / 2 \mathrm{Hrn}$ in $\mathrm{F} / 2 \mathrm{Tr}$ in $\mathrm{C} / 3 \mathrm{Pos} / \mathrm{Pk} \mathrm{A}-\mathrm{E} / 2 \mathrm{Hrf} / 1,2 \mathrm{Vl} / \mathrm{Br} / \mathrm{Vlc} / \mathrm{Kb}$ ]

14. (Fol. 119 bis 128) La Serenade/Drame Lirique en un Acte [Paris, 20. Juni 1899, Orchesterpartitur, Frag.; Picc/2 Fl/2 Ob/E. Hrn/2 Kl in B/Bkl in B/3 Fag/4 Hrn in F/4 Tr in C/3 $\mathrm{Pos} / \mathrm{Tb} / \mathrm{Pk} / 1,2 \mathrm{Vl}$ (geteilt)/Br/Vlc/Kb]

\section{Ausgewählte Literatur (chronologisch geordnet)}

A. Guerra y Alarcón, Isaac Albéniz, notas crítico biográficas de tan eminente pianista, Barcelona 1886 (Nachdr., Madrid 1990)

H. Collet, Albéniz et Joaquim Malats, Revista Musical Catalana 6, 1909, S. 377

J. de Marliave, Isaac Albéniz, in: Études Musicales, Paris 1917, S. 119ff.

H. Collet, Albéniz et Granados, Paris 1926 (2. Aufl; rev. Aufl. Paris 1948; Nachdr. der 2. Aufl. Paris 1982)

H. Collet, L'essor de la musique espagnole au XX siècle, Paris 1929

E. Istel, Isaac Albéniz, in: Musical Quarterly XV, 1929, S. 117ff.

A. de las Heras, Vida de Albéniz, Barcelona und Madrid 1941

V. Ruiz Albéniz, Isaac Albéniz, Madrid 1948

A. Sagardía, Isaac Albéniz, Madrid 1951

G. Laplane, Albéniz, sa vie, son oeuvre, Genf 1956

J. M. Llorens Cisteró, Notas inéditas sobre el virtuosismo de Isaac Albéniz y su producción pianística, in: Anuario Musical XIV, 1959, S. $91 \mathrm{ff}$.

C. Debussy, Monsieur Croche et autres écrits, Paris 1971, S. $244 \mathrm{ff}$. 
P. Buck Mast, Style and Structure in Iberia by Isaac Albéniz, Rochester 1974

C. Dahlhaus, Hrsg., Die Musik des 19 Jahrhunderts, in: Neues Handbuch der Musikwissenschaft, Bd. 6, Laaber 1980

M. Tricás Preckler, Hrsg., Cartas de Paul Dukas a Laura Albéniz, Barcelona 1983

V. Jankélévitch, La présence lointaine: Albéniz, Séverac, Mompou, Paris 1983

Ch. Le Bordays, Un siècle de présence espagnole dans la musique française, Diss., Paris 1984

L. Powell, Spain in the Music of the West. The $19^{\text {th }}$ Century Instrumental and Vocal Music, in: Kongreßbericht des internationalen Kongresses España en la Música de Occidente (1985), Madrid 1987, S. 199ff.

J. C. Gómez Amat, Sinfonismo y música de cámara en la España del siglo XIX, in: Kongreßbericht des internationalen Kongresses España en la Música de Occidente (1985), Madrid 1987, S. $211 \mathrm{ff.}$

E. Franco, Hrsg., Albéniz y su tiempo, Ausstellungskatalog, Barcelona 1990

I. Albéniz, Impresiones y diarios de viaje, [Tagebücher 1880-1904, Reisebericht 1880, Reisebericht durch Europa 1897] Madrid 1990

W. A. Clark, Albéniz en Leipzig y Bruselas: nuevas luces sobre una vieja historia, in: Revista de Musicología XIV, Nr. 5, 1-2, 1991, S. 213ff.

M. Falces, Albéniz en Inglaterra, una etapa oscura, in: Revista de Musicología XIV, Nr. 5, 1-2, 1991, S. 219ff.

M. Falces, El pacto de Fausto: estudio lingüístico-documental de los lieder ingleses de Albéniz. sobre poemas de F. B. Money-Coutts, Diss, Granada 1993

P. Baytelman-Dobry, Isaac Albéniz: chronological list and thematic catalog of his piano works, Diss., Warren, Mich. 1993

J. Soler, Vorwort zu Albéniz' Pepita Jiménez, Comedia Lírica en dos actos, revidiert und herausgegeben von J. Soler, Madrid 1996

J. Torres, Concentración vs. dispersión de fondos documentales. El desdichado caso de Isaac Albéniz, in: El patrimonio musical: los archivos familiares (1898-1936), Trujillo 1997, S. $55 \mathrm{ff}$.

ders., Iberia de Isaac Albéniz a través de sus manuscritos/Iberia by Isaac Albéniz through the manuscripts, Barcelona 1998 [Booklet für die Faksimiledition der Suite Iberia 


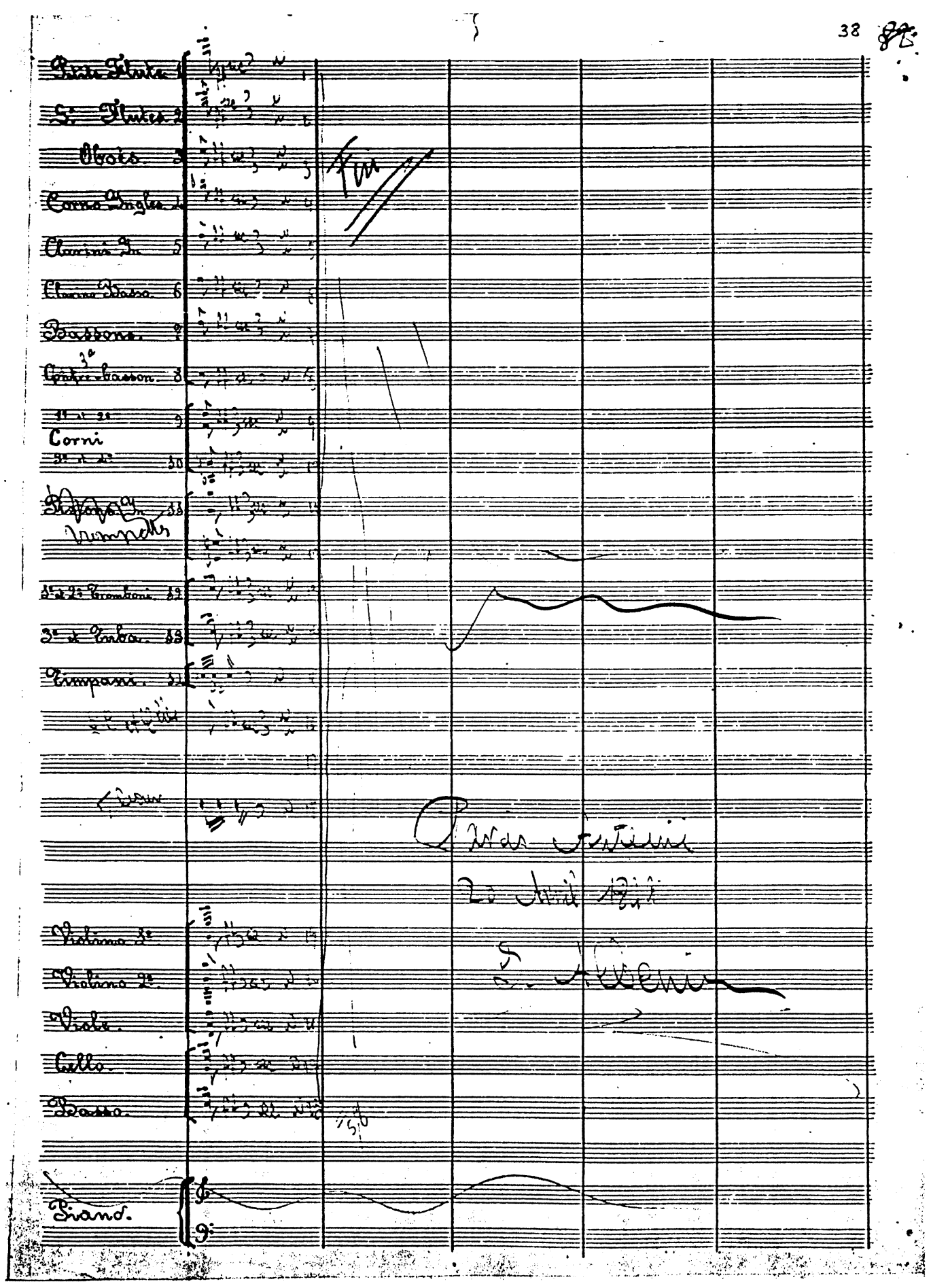

Seite aus dem Autographen der ersten Version (1899) der Suite Catalonia. [M.984, Fol. 38] 